\title{
Light Bending as a Probe of the Nature of Dark Energy
}

\author{
F. Finelli, ${ }^{1,2,3}$ M. Galaverni, ${ }^{1,4,3}$ and A. Gruppuso ${ }^{1,3}$ \\ ${ }^{1}$ INAF-IASF Bologna, via Gobetti 101, I-40129 Bologna - Italy \\ ${ }^{2}$ INAF, Osservatorio Astronomico di Bologna, via Ranzani 1, I-40127 Bologna - Italy \\ ${ }^{3}$ INFN, Sezione di Bologna, Via Irnerio 46, I-40126 Bologna - Italy \\ ${ }^{4}$ Dipartimento di Fisica, Università di Ferrara, via Saragat 1, I-44100 Ferrara - Italy
}

(Dated: April 11, 2018)

\begin{abstract}
We study the bending of light for static spherically symmetric (SSS) space-times which include a dark energy contribution. Geometric dark energy models generically predict a correction to the Einstein angle written in terms of the distance to the closest approach, whereas a cosmological constant $\Lambda$ does not. While dark energy is associated with a repulsive force in cosmological context, its effect on null geodesics in SSS space-times can be attractive as for the Newtonian term. This dark energy contribution may be not negligible with respect to the Einstein prediction in lensing involving clusters of galaxies. Strong lensing may therefore be useful to distinguish $\Lambda$ from other dark energy models.
\end{abstract}

PACS numbers: 95.36.+x,95.30.Sf,04.50.+h

\section{INTRODUCTION}

It is still unclear what drives the universe into acceleration recently. While a cosmological constant $\Lambda$ is the simplest explanation, its value seems completely at odd with the naive estimate of the vacuum energy due to quantum effects.

An alternative to $\Lambda$ is obtained considering a dynamical degree of freedom added to the primordial soup. Since dynamical dark energy ( $\mathrm{dDE}$ ) varies in time and space, its fluctuations are potentially important in order to distinguish it from $\Lambda$ [1]. Another possibility for the explanation of the present acceleration of the universe is given by the geometry itself, through a modification of Einstein gravity at large distances 2]: these are known as geometric dark energy (gDE) models. A non vanishing mass for the graviton is among these possibilities [3].

Cosmological observations, such as those coming from Supernovae, cosmic microwave background (CMB) anisotropies and large scale structure (LSS), have not been able to discriminate among dDE models yet (see [4] for updated constraints and forecasts). It is therefore important to explore observational tests at astrophysical level for objects which are detached from the cosmological expansion.

\section{DEFLECTION OF LIGHT}

We shall consider a SSS metric in terms of the physical radius $r$

$$
d s^{2}=-B(r) d t^{2}+A(r) d r^{2}+r^{2} d \Omega^{2} .
$$

Such a metric with

$$
B(r)=A^{-1}(r)=1-\frac{2 G M}{r}-\frac{\Lambda}{3} r^{2},
$$

describes the Schwarzschild-de Sitter (SdS) space-time, the vacuum solution of Einstein equations in presence of

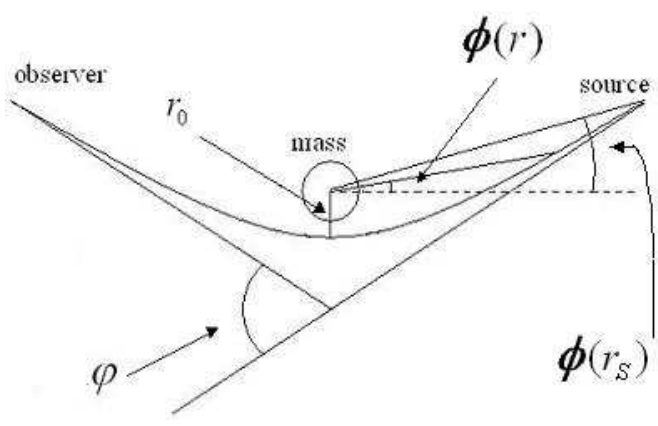

FIG. 1: Deflection of Light

a cosmological constant $\Lambda$ (in $c=1$ units, where $c$ is the velocity of light). With the above metric, the classic general relativistic tests can be computed in analogy with the Schwarzschild (henceforth $\mathrm{S}$ ) textbook case 5]. We shall restrict here to light bending, leaving other results for elsewhere [6, 7].

The textbook deflection angle for a photon in a SSS metric can be easily extended by keeping into account the finite distance $r$ at which the object is located as (see Fig (1)

$$
\begin{aligned}
\varphi & =2\left|\phi\left(r_{0}\right)-\phi(r)\right|-\pi+2 \arcsin \left(\frac{r_{0}}{r}\right), \\
& \equiv 2 I\left(r, r_{0}\right)-\pi+2 \arcsin \left(\frac{r_{0}}{r}\right),
\end{aligned}
$$

with $r_{0}$ representing the minimal distance between the geodesic of the light and the lens [8, 9] and $I\left(r, r_{0}\right)$ given by:

$$
\begin{aligned}
I\left(r, r_{0}\right) & =\int_{r_{0}}^{r} \frac{A^{1 / 2}\left(r^{\prime}\right)}{r^{\prime}}\left[\left(\frac{r^{\prime}}{r_{0}}\right)^{2} \frac{B\left(r_{0}\right)}{B\left(r^{\prime}\right)}-1\right]^{-1 / 2} d r^{\prime} \\
& \equiv \int_{r_{0}}^{r} \mathcal{I}\left(r^{\prime}, r_{0}\right) d r^{\prime} .
\end{aligned}
$$

In the textbook treatment [5], both the distances of the 
observer and the source from the lens are much greater than $r_{0}$ and the upper limit of integration can be taken as $\infty$. For DE metrics with non-asymptotically flat terms, it is safe to keep finite the upper limit of integration.

By inserting the $\operatorname{SdS}$ metric in $\mathcal{I}\left(r, r_{0}\right)$, it is easy to check that the terms including $\Lambda$ cancel out! This cancellation is due to the particular form of the nonasymptotically flat terms of the SdS metric and physically means that $\Lambda$ is truly a potential offset for a massless particle (see below). The light bending angle is therefore $4 G M / r_{0}$ even in presence of $\Lambda[10,11]$, although all the other tests and kinematical quantities differ from the $\mathrm{S}$ case [6, 7].

\section{GEOMETRIC DARK ENERGY STATIC SPHERICALLY SYMMETRIC METRICS}

In order to study the contribution of DE to light bending, we need physically motivated SSS metrics which differ from the SdS case. As an example, we consider SSS metrics parametrized by:

$$
\begin{aligned}
B(r) & =C-\frac{D}{r}-\gamma_{2} r^{\alpha} \\
A^{-1}(r) & =C-\frac{D}{r}-\gamma_{1} r^{\alpha} .
\end{aligned}
$$

This parametrization covers SSS metrics of well studied gDE models:

- $C=1, D=2 G M, \alpha=3 / 2, \gamma_{1}=$ $3 \gamma_{2} / 2=-m_{g}^{2} \sqrt{2 G M / 13}$ [12] corresponds to the non-perturbative solution found by Vainshtein (V) for a massive graviton [13] with mass $m_{g}$. Massive gravity (MG) is an alternative to a cosmological constant [3];

- $C=1+3 G M \gamma_{1}, D=2 G M+3 G^{2} M^{2} \gamma_{1}, \alpha=$ $1, \gamma_{1}=\gamma_{2}=\gamma$ corresponds to the general SSS in conformal gravity [14] (see also [15] for linear correction to the Newtonian potential) . Conformal gravity contains the SdS solution without adding any cosmological constant to the Weyl action, but includes terms linear in $r$ as well. Considering $C \simeq$ 1 and $D \simeq 2 G M$ is a good approximation for the values of $\gamma$ we are interested in;

- $C=1, D=2 G M, \alpha=1 / 2, \gamma_{1}=\gamma_{2} / 2=$ $\sqrt{G M / r_{c}^{2}}$ corresponds to the self-accelerating branch [16] of the brane induced gravity DvaliGabadadze-Porrati (DGP) model [17]. $r_{c}$ is the crossover scale beyond which gravity becomes fivedimensional.

In the parametrization (5) we have omitted a possible $r^{2}$ term, since we have already shown that it does not contribute to light bending. For $\alpha=1$ the SSS metric is valid till to the particle horizon radius $r_{H} \sim \gamma^{-1}$ (for the $\mathrm{SdS}$ metric this radius is $\left.(3 / \Lambda)^{1 / 2}\right)$. In the other two cases the metric is roughly limited by the so-called $\mathrm{V}$ radius $r_{V}$, which denotes the scale at which the Newtonian term becomes comparable to the non-asymptotically flat term, i.e. $r_{V} \sim(G M / \gamma)^{1 /(1+\alpha)}$ with $\gamma^{-1}=\min \left\{\gamma_{1}^{-1}, \gamma_{2}^{-1}\right\}$. At this scale we expect deviations from Einstein gravity.

We now expand the integrand considering the DE terms in Eq. (4) as second order with respect to the Newtonian correction, and, defining $I\left(r, r_{0}\right)=I_{\mathrm{E}}\left(r, r_{0}\right)+I_{\mathrm{DE}}\left(r, r_{0}\right)$ we obtain:

$$
\begin{array}{r}
I_{\mathrm{E}}\left(r, r_{0}\right)=\int_{r_{0}}^{r} \frac{d r^{\prime}}{r^{\prime}}\left[\left(\frac{r^{\prime}}{r_{0}}\right)^{2}-1\right]^{-1 / 2}\left[1+\frac{G M}{r^{\prime}}+\frac{G M r^{\prime}}{r_{0}\left(r^{\prime}+r_{0}\right)}+\frac{3(G M)^{2}}{2 r^{\prime 2}}+\frac{3(G M)^{2}}{r_{0}\left(r^{\prime}+r_{0}\right)}+\frac{3(G M)^{2} r^{\prime 2}}{2 r_{0}^{2}\left(r^{\prime}+r_{0}\right)^{2}}\right], \\
I_{\mathrm{DE}}\left(r, r_{0}\right)=\int_{r_{0}}^{r} \frac{d r^{\prime}}{r^{\prime}}\left[\left(\frac{r^{\prime}}{r_{0}}\right)^{2}-1\right]^{-1 / 2} \frac{\left[\left(\gamma_{1}-\gamma_{2}\right) r^{\prime(\alpha+2)}+\gamma_{2} r^{\prime 2} r_{0}^{\alpha}-\gamma_{1} r^{\prime \alpha} r_{0}^{2}\right]}{2\left(r^{\prime 2}-r_{0}^{2}\right)} .
\end{array}
$$

It is possible to analitically compute both the terms in Eqs. (617):

$$
\begin{gathered}
I_{\mathrm{E}}\left(r, r_{0}\right)=\frac{\pi}{2}-\arcsin \left(\frac{r_{0}}{r}\right)+\frac{G M}{r_{0}}\left(1-\frac{r_{0}}{r}\right)^{1 / 2}\left[\left(1+\frac{r_{0}}{r}\right)^{1 / 2}+\left(1+\frac{r_{0}}{r}\right)^{-1 / 2}\right]+\mathcal{O}\left(\frac{G^{2} M^{2}}{r_{0}^{2}}\right), \\
I_{\mathrm{DE}}\left(r, r_{0}\right)=\frac{r_{0}^{\alpha}}{2} I_{\alpha}\left(\frac{r}{r_{0}}\right)\left(\gamma_{1}-\alpha \gamma_{2}\right)+\gamma_{2} \frac{r_{0}^{\alpha}}{2} \frac{\left(r / r_{0}\right)^{\alpha}-1}{\sqrt{\left(r / r_{0}\right)^{2}-1}},
\end{gathered}
$$

with

$$
I_{\alpha}(y)=\int_{1}^{y} d x \frac{x^{\alpha-1}}{\sqrt{x^{2}-1}}=\frac{\sqrt{\pi}}{2} \frac{\Gamma\left(\frac{1-\alpha}{2}\right)}{\Gamma\left(1-\frac{\alpha}{2}\right)}+\frac{y^{\alpha-1}}{\alpha-1}{ }_{2} F_{1}\left(\frac{1}{2}, \frac{1-\alpha}{2}, \frac{3-\alpha}{2}, \frac{1}{y^{2}}\right) .
$$

It is important to note that the physical structure of gDE metric kill the first term in Eq. (9) for all the three values $\alpha$. Of course, the type of DE contribution 
to the deflection angle is not of the parametrized postNewtonian (PPN) form [5], since the correction in the metric coefficient is not of the PPN form. Note that our non-vanishing result for the DGP model corrects previous claims in the literature [18] and agrees with previous result for Weyl gravity when $r \rightarrow \infty$ [19]. A non-negligible DE contribution to the total deflection angle is expected for large virialized objects, i.e in the case of clusters of galaxies.

In the following, we split the Einstein term $\varphi_{E}$ from the dark energy correction $\Delta \varphi$ to the total bending angle $\varphi$. Therefore, the DE contribution has to be compared with the S term assuming $r \rightarrow \infty$ (up to the second order [20]):

$$
\varphi_{\mathrm{E}}=\varphi^{(I)}+\varphi^{(I I)}=
$$

$$
=\frac{4 G M}{r_{0}}+2\left(\frac{15 \pi}{8}-2\right)\left(\frac{G M}{r_{0}}\right)^{2}
$$

This comparison is shown in Table \

The sign of the DE contribution can be understood by the one dimensional motion for the photon:

$$
\frac{1}{2}\left(\frac{d r}{d \lambda}\right)^{2}+V_{\text {eff }}(r)=\frac{1}{2}
$$

with $d \lambda \equiv r^{2} d \varphi / J$ and

$$
V_{\mathrm{eff}}(r)=\frac{J^{2}}{2 r^{2}}\left[\frac{1}{A(r)}+\frac{\left(\gamma_{1}-\gamma_{2}\right) r^{\alpha}}{J^{2} B(r)}\right]=\frac{J^{2}}{2 r^{2}}\left[C-\frac{D}{r}-\gamma_{1} r^{\alpha}+\frac{\left(\gamma_{1}-\gamma_{2}\right) r^{\alpha}}{J^{2}\left(C-\frac{D}{r}-\gamma_{2} r^{\alpha}\right)}\right] .
$$

This potential leads to the following force $F$ on a photon:

$$
\frac{F(r)}{J^{2}}=-\frac{d V_{\mathrm{eff}}}{J^{2} d r}=\frac{C}{r^{3}}-\frac{3 D}{2 r^{4}}+\frac{\gamma_{1}}{2}(\alpha-2) r^{\alpha-3}+\left(\gamma_{2}-\gamma_{1}\right) \frac{r^{\alpha}}{2 J^{2}\left(C-\frac{D}{r}-\gamma_{2} r^{\alpha}\right)^{2}}\left[\alpha \frac{C}{r}-\frac{D}{2}(1+\alpha)\right]
$$

From the above it is again clear how $\Lambda$ acts as a null force on the photon (in agreement with the null contribution in light bending [21]). When $\alpha<2$, in addition to the standard terms, the third term acts as an attractive force for $\gamma_{1}>0$. We also note that the sign of the fourth term is model dependent [22]. Although DE in cosmology is associated to a repulsive force (which accelerates the expansion), in a static configuration it may add to the Newtonian mass term in deflecting light, explaining the positive contribution which we find in Eq. (9).

\begin{tabular}{|c|c|c|c|c|c|}
\hline & $\varphi^{(I)}$ & $\varphi^{(I I)}$ & $\Delta \varphi_{\mathrm{MG}}$ & $\Delta \varphi_{\text {Weyl }}$ & $\Delta \varphi_{\text {DGP }}$ \\
\hline Galaxy $\left(10^{11} M_{\odot}\right)$ & $2.0 \times 10^{-6}$ & $1.9 \times 10^{-12}$ & $-4.6 \times 10^{-11}$ & $1.0 \times 10^{-6}$ & $3.9 \times 10^{-9}$ \\
\hline Galaxy Group $\left(10^{13} M_{\odot}\right)$ & $1.9 \times 10^{-5}$ & $1.9 \times 10^{-10}$ & $-7.2 \times 10^{-9}$ & $1.0 \times 10^{-5}$ & $2.3 \times 10^{-7}$ \\
\hline Cluster $\left(10^{15} M_{\odot}\right)$ & $1.8 \times 10^{-4}$ & $1.8 \times 10^{-8}$ & $-1.1 \times 10^{-6}$ & $1.0 \times 10^{-4}$ & $6.6 \times 10^{-6}$ \\
\hline
\end{tabular}

TABLE I: Expected deviations of the three theories considered from Einstein theory. The parameters used are the following: $m_{g}=10^{-31} \mathrm{eV}, \gamma^{-1}=10 \mathrm{Gpc}, r_{c}=5 \mathrm{Gpc}$. The distance of closest approach are $r_{0}=10,10^{2}, 10^{3} \mathrm{Kpc}$ for galaxy, galaxy groups, clusters, respectively. The upper limits of integration have been taken as $r_{V}$ for $\alpha=1 / 2,3 / 2$ and $\infty$ for Weyl gravity. We remind that the Hubble distance $1 / H_{0}$ is $\sim 4 \mathrm{Gpc}$ for $H_{0}=72 \mathrm{~km} \mathrm{~s}^{-1} \mathrm{Mpc}^{-1}$. As written also in the text, the contribution $\Delta \varphi_{\mathrm{BV}}$ coming from the region beyond $r_{V}$ is taken into account only in DGP model [16, 18] and it is given by $\Delta \varphi_{\mathrm{BV}}=$ $\left(G\left(r_{\max }\right)-G\left(r_{V}\right)\right) 2 G M / r_{0}$ where $G(r)=\left(1-\left(r_{0} / r\right)^{2}\right)^{1 / 2}\left(1+f(\omega)\left(r_{0} / r\right)\right)\left(1+\left(r_{0} / r\right)\right)^{-1}$ with $f(\omega)=(\omega+1) /(2 \omega+3)$. The parameter $\omega=-3 r_{c} H_{0}$ is set to $-15 / 4$ and $r_{\max }=1 \mathrm{Gpc}$.

We note that the validity of the SSS metrics for $\alpha=$ $1 / 2,3 / 2$ up to the $\mathrm{V}$ radius - and not up to the particle horizon - can be an important limitation to the applicability of our findings. In the DGP (massive gravity) model with $r_{c}=5 \mathrm{Gpc}\left(m_{g} \sim 10^{-32} \mathrm{eV}\right)$, the $\mathrm{V}$ radius for the Sun is $3.2 \times 10^{18} \mathrm{~m}\left(7.5 \times 10^{20} \mathrm{~m}\right)$, and therefore much larger than the size of the solar system $\sim 6 \times 10^{12}$ $\mathrm{m}$ (taken as the size of the Pluto orbit). For clusters with mass $\sim 10^{15} M_{\odot}$, instead, the $\mathrm{V}$ radius is $10 \mathrm{Mpc}$ for DGP and $24 \mathrm{Mpc}$ for MG (with the same parameters used above): such radii are remarkably close to the intercluster distance, i.e. $\mathcal{O}(10) \mathrm{Mpc}$. It is then clear that an understanding of the SSS metrics beyond the $\mathrm{V}$ scale (the so-called matching problem [12]) is needed for a 


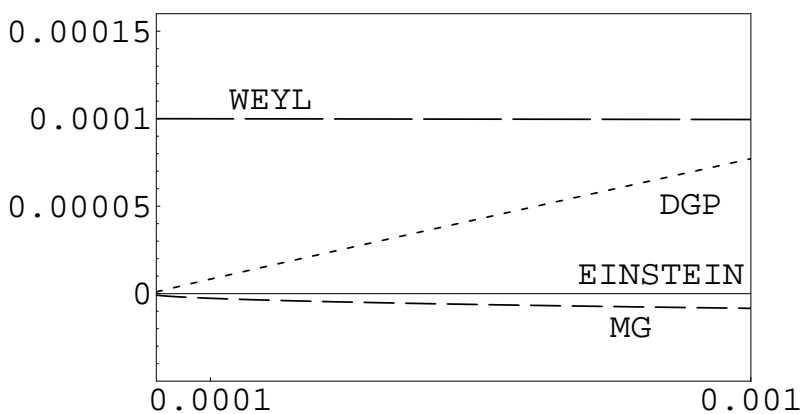

FIG. 2: Dark Energy contribution $\Delta \varphi_{\mathrm{DE}}$ to the bending angle (in radiants) as a function of $G M / r_{0}$. The solid line $\left(\Delta \varphi_{\mathrm{DE}}=\right.$ $0)$ is the Einstein prediction for $\Lambda$; the dotted, long-dashed and dashed are the DGP, Weyl and MG models, respectively, with $r_{0}=1 \mathrm{Mpc}$ (the other parameters are those of Table I). Note that $\gamma$ is $M$ independent [14].

quantitatively exact calculation of light bending in these models. While the MG metric is not known beyond $r_{V}$, the DGP metric beyond $r_{V}$ admits a scalar-tensor description [16, 18], which we use for the values reported in Table I (see the caption) and in Fig 2 where we show the prediction of the considered models compared with Einstein theory as function of $G M / r_{0}$.

\section{DISCUSSIONS AND CONCLUSIONS}

We have discussed light bending in SSS with DE. The importance of general relativistic tests, such as the perihelion precession, has been already emphasized for the DGP model [16, 23], while little attention was previously paid to light bending. These tests are complementary to the observational signatures of dark energy in cosmological context, mainly based on the behaviour of perturbations. In cosmology, an important difference between $\Lambda$ and $\mathrm{dDE}$ or gDE is the presence of DE perturbations in the latter case, which are at least gravitationally coupled to the other types of matter. Such DE perturbations are therefore a key point to distinguish $\Lambda$ from $\mathrm{dDE}$ or gDE in CMB and LSS, and sometimes may become so important to strongly constrain models [1, 24, 25] with respect to what Supernovae data can do.

In this article we have shown that in objects which have detached from the expansion of the universe, $\Lambda$ may be distinguishable from other DE models through the bending of light. In order to link our findings with observations, we should insert $\varphi$ in the lens equation, e.g. [26] :

$$
\theta-\beta=\frac{d_{S L}}{d_{O S}} \varphi
$$

where $\theta$ and $\beta$ are the angular positions of the image and of the source measured respect to the line from the observer to the lens; $d_{L S}$ and $d_{O S}$ are the angular diameter distances between the lens and the source and between the observer and the source, respectively. On considering for simplicity alignment between the lens and the source, an Einstein ring forms with angle $\theta_{E}=\theta(\beta=0)$. From our results, $\theta_{E}$ is affected by both the non-perturbative SSS potential around the lens $\left(\varphi \neq 4 G M / r_{0}\right.$ if $\left.\mathrm{DE} \neq \Lambda\right)$ and the cosmology of a given model. The gDE corrections to the Einstein deflection angle for clusters in Eq. (9) are as important as the cosmology for an observable as $\theta_{E}$. The differential of $\theta_{E}$ is

$$
\frac{\Delta \theta_{E}}{\theta_{E}}=\frac{\Delta \varphi}{\varphi}+\Delta \ln \frac{d_{S L}}{d_{O S}}
$$

which reveals how cosmological information is encoded just in the second term to the right. By considering the cosmology of the DGP model for instance [18], one finds that the second term is $\sim-0.06\left(\Delta \Omega_{\mathrm{M}} / \Omega_{\mathrm{M}}\right)+\Delta H_{0} / H_{0}$ for a source and a lens located at $z=1$ and $z=0.3$, respectively $\left(\Omega_{\mathrm{M}} \sim 0.3\right.$ and $H_{0}$ are the present matter density and Hubble parameter, respectively). On considering the uncertainties on the cosmological parameters of the order of percent, this simple quantitative example shows how the corrections to the Einstein deflection angle we have found in Table I should be taken into account in the study of strong lensing by clusters.

We believe that results similar to what we have found here for gDE models, might occur for dDE scenarios as well, in which the non-asymptotically flat term is due to the non-perturbative clumping of DE into objects detached from the cosmological expansion. However, dDE models may be less predictive than gDE models: $\mathrm{gDE}$ contain the same number of parameters of $\Lambda \mathrm{CDM}$, while dDE may need more. Let us end on noting that some of the gDE models considered here may have serious theoretical issues [27, 28], whose resolution clearly go beyond the present project. However, the main result in Eq. (9) of this paper remains valid: models alternative to general relativity with a cosmological constant predict a correction to the Einstein angle, which can be used to distinguish $\Lambda$ from other DE models.

Acknowledgements We wish to thank Lauro Moscardini for many discussions and suggestions on galaxies, groups and clusters. We are grateful to Robert $\mathrm{R}$. Caldwell and one of the anonymous referees for valuable comments on this project. FF and MG are partially supported by INFN IS PD51; FF and and AG are partially supported by INFN IS BO11. The authors thank the Galileo Galilei Institute for Theoretical Physics for the hospitality and the INFN for partial support during the developments of this project. 
[1] R. R. Caldwell, R. Dave and P. J. Steinhardt, Phys. Rev. Lett. 80 (1998) 1582.

[2] C. Deffayet, G. R. Dvali and G. Gabadadze, Phys. Rev. D 65 (2002) 044023.

[3] G. Gabadadze and A. Gruzinov, Phys. Rev. D 72 (2005) 124007.

[4] A. Upadhye, M. Ishak and P. J. Steinhardt, Phys. Rev. D 72 (2005) 063501.

[5] S. Weinberg, Gravitation and Cosmology: Principles and Applications of the General Theory of Relativity, Wiley, New York, 1972.

[6] M. Galaverni, Test Relativistici per Modelli di Energia Oscura, Laurea Thesis, University of Bologna, 2005 (unpublished).

[7] F. Finelli, M. Galaverni, A. Gruppuso, in preparation (2007).

[8] For semplicity we consider here the object and the observer at the same distance from the lens.

[9] We choose to present our results in terms of the distance of closest approach $r_{0}$, as in [5]. Another convention is to write the results in terms of the impact parameter $b$ [29], which has the advantage of being an invariant of the light ray with respect to $r_{0}$. For the Schwarzschild metric the relation between these two quantities is [29]:

$$
r_{0}=\frac{2 b}{\sqrt{3}} \cos \left[\frac{1}{3} \arccos \left(-\frac{3 \sqrt{3} G M}{b}\right)\right]
$$

which is given as a root of cubic polynomial. For $G M \ll$ $b, r_{0}=b(1+\mathcal{O}(G M / b))$ and therefore can be identified at leading order. The differences of the Einstein angle expressed in form $r_{0}$ or $b$ are therefore in the next-toleading order terms, i.e. $\mathcal{O}\left(G^{2} M^{2} / r_{0}^{2}\right)$ or $\mathcal{O}\left(G^{2} M^{2} / b^{2}\right)$ (see fourth reference of [20]).

For SdS the impact parameter $b$ is not directly $J / E(J$ and $E$ being the angular momentum and the energy, respectively). The relation between $r_{0}$ and $J / E$ (the latter being the invariant of the light ray) for $\mathrm{SdS}$ is:

$$
r_{0}=\frac{2 J}{\sqrt{3 E^{2}+\Lambda J^{2}}} \cos \left[\frac{1}{3} \arccos \left(-\frac{3 \sqrt{3 E^{2}+\Lambda J^{2}} G M}{J}\right)\right] .
$$

Again $r_{0} \simeq J / E$ in the SdS case at leading order for $G M E / J \ll 1, \Lambda J^{2} / E^{2} \ll 1$.
[10] J. N. Islam, Phys. Lett. A 97 (1983) 239.

[11] Note that the light bending in SdS written in terms of $J / E$ (rather than $r_{0}$ ) contains $\Lambda$ because of the relation between $r_{0}$ and $J / E$ in [9].

[12] T. Damour, I. I. Kogan and A. Papazoglou, Phys. Rev. D 67 (2003) 064009.

[13] A. I. Vainshtein, Phys. Lett. B 39, 393 (1972).

[14] P. D. Mannheim and D. Kazanas, Astrophys. J. 342 (1989) 635.

[15] S. L. Dubovsky, P. G. Tinyakov and I. I. Tkachev, Phys. Rev. D 72 (2005) 084011.

[16] A. Lue and G. Starkman, Phys. Rev. D 67 (2003) 064002.

[17] G. R. Dvali, G. Gabadadze and M. Porrati, Phys. Lett. B 485 (2000) 208.

[18] A. Lue, Phys. Rept. 423 (2006) 1.

[19] A. Edery and M. B. Paranjape, Phys. Rev. D 58 (1998) 024011; S. Pireaux, Class. Quant. Grav. 21, 1897 (2004).

[20] R. Epstein and I. I. Shapiro, Phys. Rev. D 22 (1980) 2947; E. Fischbach and B. S. Freeman, Phys. Rev. D 22 (1980) 2950; K. S. Virbhadra, D. Narasimha and S.M. Chitre, A. \& A. 337 (1998) 1; J. Bodenner and C. M. Will, Am. J. Phys. 71, 770 (2003).

[21] Note that this is not true for a massive particle. $\Lambda$ induces a force on a test mass, for example producing an additional shift in the perihelion precession [6, 30].

[22] In the models studied here this fourth term is $\propto \gamma_{2}(1-\alpha)$ : for $\gamma_{2}>0$ it is an attractive (repulsive) force for $\alpha=1 / 2$ $(3 / 2)$. It is zero for conformal gravity, $\alpha=1$.

[23] G. Dvali, A. Gruzinov and M. Zaldarriaga, Phys. Rev. D 68 (2003) 024012.

[24] D. Carturan and F. Finelli, Phys. Rev. D 68 (2003) 103501; L. Amendola, F. Finelli, C. Burigana, D. Carturan, JCAP 07 (2003) 005.

[25] K. Koyama and R. Maartens, JCAP 01 (2006) 016.

[26] P. Schneider, J. Ehlers, E. E. Falco, Gravitational Lenses, Springer, 1999.

[27] D. Boulware and S. Deser, Phys. Rev. D 6 (1972) 3368.

[28] D. Gorbunov, K. Koyama and S. Sibiryakov, Phys. Rev. D 73 (2006) 044016;

[29] R. Wald, General Relativity, Chicago, University Press, 1984.

[30] E. L. Wright, arXiv:astro-ph/9805292 